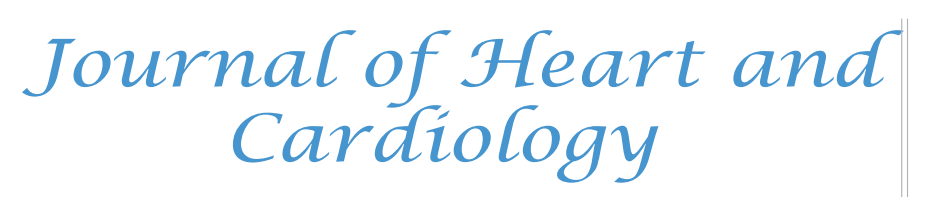

\title{
A 25 Year Review of Combined Cardiac and Renal Transplant Outcomes in Patients with End Stage Cardiac Failure on Renal Replacement Therapy. A Single Center Experience
}

\author{
Chris Anthony $^{1 *}$, Andrew Jabbour ${ }^{1}$, Eugene Kotlyar ${ }^{1}$, Anne M. Keogh ${ }^{1}$, Christopher S. Hayward ${ }^{1}$, Peter \\ S. Macdonald ${ }^{2}$, Jacob Sevastos ${ }^{3}$ \\ ${ }^{1}$ Department of Cardiology, Heart Failure and Transplant St Vincent's Hospital, Sydney, New South Wales, Australia \\ ${ }^{2}$ Department of Cardiac and Thoracic Surgery, St Vincent's Hospital, Sydney, New South Wales, Australia \\ ${ }^{3}$ Department of Renal Medicine and Transplantation St Vincent's Hospital, Sydney, New South Wales, Australia
}

*Corresponding author: Chris Anthony, MBBS, Department of Cardiology, Heart Failure and Transplant St Vincent's Hospital, 1/569 George Street, Sydney-2000, New South Wales, Australia, Tel: 0061424132549; E-mail: chris007_7@hotmail.com, canthony@stvincents.com.au

Citation: Anthony, C., et al. A 25 Year Review of Combined Cardiac and Renal Transplant Outcomes in Patients with End Stage Cardiac Failure on Renal Replacement Therapy. A Single Center Experience. (2016) J Heart Cardiol 2(2): 59-67.

Received Date: February 08, 2016; Accepted Date: July 12, 2016; Published Date: July 15, 2016

\begin{abstract}
Background: Combined heart and kidney transplantation has been shown to be a viable option for patients who have concurrent end stage cardiac and renal failure. However there is limited long term survival data that compares the outcomes of patients undergoing combined heart-kidney transplantation to patients undergoing solitary cardiac transplantation. There is also limited data on patients with end stage cardiac failure who are on concurrent renal replacement therapy prior to organ transplantation and their outcomes. This study reviews the short and long term outcomes of combined heart kidney transplantation over a 25 year period in comparison to solitary cardiac transplantation in a majority of patients undergoing renal replacement therapy at time of transplant listing. Methods and Results: In total there were 16 patients who underwent combined heart and kidney transplantation in the period between October 1990 and June 2014 (including heart and kidney re-transplantation) with 14 patients (87.5\%) on renal replacement therapy at time of combined procedure. They were listed for combined heart and kidney transplantation as they fulfilled our institution's criteria for irreversible end-stage heart and kidney failure. Retrospective review of patient data from the transplant database, patient case notes and post-mortem reports were carried out. Statistical analysis was then performed on key patient demographics alongside actuarial survival analysis, which were then graphically annotated. IRB approval was obtained and informed consent from patients was also obtained.

The mean (SD) recipient age was 42 (13) years and there were 3 females. Dilated cardiomyopathy was the most common primary cardiac pathology (50\%) whilst ischemic nephrosclerosis $(25 \%)$ and glomerulonephritis $(25 \%)$ were the most common primary renal pathologies. Most patients experienced NYHA class IV symptoms $(62.5 \%)$. The average wait time to transplantation at our institution was 12 months.

There was no operative mortality. The cumulative 1 year survival in the combined transplant group was 0.75 with 4 out of 16 mortalities within the first year $(25 \%)$. In comparison the cumulative 1 year survival of the heart only transplant group was 0.86 with 116 mortalities within the first year over a 25 year period.

Cumulative survival at $5,10,15$ and 25 years for the combined transplant group was $0.69,0.55,0.437$ and 0.437 respectively. In comparison cumulative survival of the heart only transplant group at the $5,10,15$ and 25 year mark was $0.76,0.59,0.45$ and 0.23 respectively.

The incidence of cardiac rejection episodes in the study time was 9 out of $16(56 \%)$ versus $3 / 16(19 \%)$ who had renal rejection. In the study period there was 1 death out of 7 deaths due to dual graft failure.

Conclusions: Combined sequential cardiac and renal transplantation has good short- and long-term outcomes for patients with coexisting end stage cardiac and renal failure. At the ten year mark actuarial survival for combined heart and kidney transplantation is equivalent to cardiac transplantation alone.
\end{abstract}

Copyrights: (C) 2016 Chris Anthony. This is an Open access article distributed under the terms of Creative Commons Attribution 4.0 International License. 
Keywords: Combined Heart Kidney Transplant; Heart Failure; Renal Failure; Cardiorenal Syndrome

\section{Introduction}

Chronic kidney disease in the context of cardiac transplantation is a significant contributor to increased morbidity and mortality ${ }^{[1,2]}$. In addition there is established evidence that between $30-40 \%$ of patients admitted to hospital with heart failure have concurrent renal impairment ${ }^{[3,4]}$ with significantly worse morbidity and mortality outcomes ${ }^{[3,5-10]}$. Since the initial report of simultaneous heart-kidney transplantation in 1978, combined solid-organ transplantation has evolved into a viable option for patients with end stage cardiac and renal failure ${ }^{[11-35]}$. This is especially important in the context of patients with end stage cardiac failure who are on renal replacement therapy, where solitary cardiac transplant alone may be contraindicated. We report our experience with 16 patients over a 25 year period 14 of whom were on concurrent renal replacement therapy which we believe is the longest retrospective review of patients to have undergone simultaneous combined heart and kidney transplantation and compared their outcomes to the outcomes of patients undergoing solitary heart transplantation at our institution.

\section{Methods}

A retrospective observational study was undertaken. During the period between October 1990 and June 2014, a total of 16 patients underwent combined heart and kidney transplantation at our institution. In the period between Feb 1984 and May 2014 a total of 866 patients underwent orthotropic heart transplantation at our institution.

A retrospective review of medical, surgical and intensive care records and the transplant database was completed on these 16 patients. The records were reviewed for patient demographics, co-morbidities, etiology of cardiac and renal failure, baseline cardiac and renal function, renal replacement requirement and duration of renal replacement prior to transplant, right heart study pre transplant, time on the transplant waiting list, peri-transplant acute and long term complications, rejection events, graft function and long term mortality outcomes. These results were documented and tabulated and patients numbered accordingly 1-16 in all tables (Table 1-4). Data was summarized using means \pm standard deviation for numeric variables and percents and counts for categorical variables. (Table 1,2)

Table 1: Patient Characteristics Pre Transplantation

\begin{tabular}{|c|c|c|c|c|c|c|c|c|c|c|}
\hline Patient & Age & Sex & $\begin{array}{l}\text { Co- } \\
\text { morbidities }\end{array}$ & $\begin{array}{l}\text { Etiology of Renal } \\
\text { Dysfunction }\end{array}$ & $\begin{array}{l}\text { Kidney } \\
\text { function } \\
\text { Creatinine } \\
\text { mmol/l }\end{array}$ & $\begin{array}{l}\text { Duration } \\
\text { of Renal } \\
\text { Replacement } \\
\text { Therapy Prior } \\
\text { to Transplant } \\
\text { or Post Trans- } \\
\text { plantation } \\
\text { Months }\end{array}$ & $\begin{array}{l}\text { Etiology of } \\
\text { Cardiac Dys- } \\
\text { function }\end{array}$ & EF & $\begin{array}{l}\text { ECMO/ } \\
\text { LVAD } \\
\text { prior to } \\
\text { Tx }\end{array}$ & $\begin{array}{l}\text { Right } \\
\text { Heart } \\
\text { Catheter } \\
\text {-Pulmo- } \\
\text { nary Artery } \\
\text { pressure } \\
\text { mmHg }\end{array}$ \\
\hline 1 & 61 & $\mathrm{M}$ & $\begin{array}{l}\text { HPT, Dyslip- } \\
\text { idemia }\end{array}$ & $\begin{array}{l}\text { Post Infection } \\
\text { GN, Ischemic } \\
\text { nephrosclerosis }\end{array}$ & 280 & $\begin{array}{l}1 \text { month HD } \\
\text { post Tx }\end{array}$ & $\begin{array}{l}\text { RF/Valvular } \\
\mathrm{CM}+\mathrm{IHD}\end{array}$ & $20 \%$ & IABP & 50 \\
\hline 2 & 23 & $\mathrm{~F}$ & $\begin{array}{l}\text { WilmsTu- } \\
\text { mors, CKD, } \\
\text { Aniridia }\end{array}$ & $\begin{array}{l}\text { WAGR syndrome- } \\
\text { Bilateral Wilms } \\
\text { Tumors- Right } \\
\text { nephrectomy Left } \\
\text { partial nephrec- } \\
\text { tomy: clq GN in } \\
\text { remaining kidney }\end{array}$ & Haemodialysis & $\begin{array}{l}60 \text { months pre } \\
\text { transplant }\end{array}$ & $\begin{array}{l}\text { Anthracycline } \\
\text { induced car- } \\
\text { diomyopathy. }\end{array}$ & $10 \%$ & IABP & 45 \\
\hline 3 & 52 & M & $\begin{array}{l}\text { Hyperten- } \\
\text { sion, Dys- } \\
\text { lipidemia, } \\
\text { Anemia, } \\
\text { Secondary } \\
\text { Hyperpara- } \\
\text { thyroidism }\end{array}$ & $\begin{array}{l}\text { Ischemic ne- } \\
\text { phrosclerosis } \\
+ \text {-cardiorenal } \\
\text { syndrome }\end{array}$ & Haemodialysis & $\begin{array}{l}96 \text { months pre } \\
\text { transplant }\end{array}$ & DCM & $15 \%$ & IABP & 40 \\
\hline 4 & 27 & M & DCM & $\begin{array}{l}\text { Chronic Allograft } \\
\text { nephropathy } \\
\text { (Multiple Heart } \\
\text { and Kidney Trans- } \\
\text { plantation) }\end{array}$ & $\begin{array}{l}\text { Continuous } \\
\text { Ambulatory } \\
\text { Peritoneal } \\
\text { Dialysis }\end{array}$ & $\begin{array}{l}12 \text { months pre } \\
\text { transplant }\end{array}$ & $\begin{array}{l}\text { Allograft } \\
\text { Vasculopathy } \\
\text { (Multiple } \\
\text { Heart and } \\
\text { Kidney Trans- } \\
\text { plantation) }\end{array}$ & $25 \%$ & Nil & 32 \\
\hline
\end{tabular}




\begin{tabular}{|c|c|c|c|c|c|c|c|c|c|c|}
\hline 5 & 43 & M & $\begin{array}{l}\text { CKD, HPT, } \\
\text { Dyslipidemia }\end{array}$ & MCGN type 1 & Haemodialysis & $\begin{array}{l}36 \text { months pre } \\
\text { transplant }\end{array}$ & $\begin{array}{l}\text { Viral } \\
\text { myocarditis }\end{array}$ & $20 \%$ & Nil & 13 \\
\hline 6 & 45 & M & $\begin{array}{l}\text { CKD, HPT, } \\
\text { Dyslipidemia }\end{array}$ & $\begin{array}{l}\text { Post Strep GN 1st } \\
\text { kidney tx }\end{array}$ & Haemodialysis & $\begin{array}{l}24 \text { months pre } \\
\text { transplant }\end{array}$ & $\begin{array}{l}\text { IHD/Valvular } \\
\text { CM/CABG } 86\end{array}$ & $15 \%$ & Nil & 40 \\
\hline 7 & 52 & M & HPT,CKD & IGA nephropathy & Haemodialysis & $\begin{array}{l}14 \text { months pre } \\
\text { transnplant }\end{array}$ & $\mathrm{DCM}$ & $20 \%$ & Nil & 45 \\
\hline 8 & 39 & M & DCM & $\begin{array}{l}\text { Calcineurin } \\
\text { inhibitor toxivity } \\
+/- \text { Cardiorenal } \\
\text { Syndrome }\end{array}$ & $\begin{array}{l}\text { Continuous } \\
\text { Ambulatory } \\
\text { Peritoneal } \\
\text { Dialysis }\end{array}$ & $\begin{array}{l}3 \text { months pre } \\
\text { transplant }\end{array}$ & $\begin{array}{l}\text { DCM+ } \\
\text { allograft } \\
\text { vasculopathy } \\
2^{\text {nd }} \text { heart }\end{array}$ & $15 \%$ & $\begin{array}{l}\text { ECMO } \\
\text { to TX }\end{array}$ & 47 \\
\hline 9 & 53 & M & $\begin{array}{l}\text { Polycystic } \\
\text { Kidney } \\
\text { Disease }\end{array}$ & $\begin{array}{l}\text { Polycystic Kidney } \\
\text { Disease }\end{array}$ & 180 & Nil & DCM & $30 \%$ & Nil & 42 \\
\hline 10 & 53 & M & $\mathrm{CKD} / \mathrm{DCM}$ & $\begin{array}{l}\text { Reflux } \\
\text { nephropathy }\end{array}$ & Haemodialysis & $\begin{array}{l}84 \text { months pre } \\
\text { transplant }\end{array}$ & $\mathrm{DCM}$ & $23 \%$ & Nil & 27 \\
\hline 11 & 32 & M & $\begin{array}{l}\text { IGA ne- } \\
\text { phropathy/ } \\
\text { CKD, Gout, } \\
\text { Dyslipidemia }\end{array}$ & IGA nephropathy & $\begin{array}{l}\text { Continuous } \\
\text { Ambulatory } \\
\text { Peritoneal } \\
\text { Dialysis } \\
\end{array}$ & $\begin{array}{l}36 \text { months pre } \\
\text { transplant }\end{array}$ & $\mathrm{DCM}$ & $19 \%$ & Nil & 30 \\
\hline 12 & 58 & M & $\begin{array}{l}\text { IHD/ } \\
\text { Anterior MI } \\
\text { leading to } \\
\text { Cardiac } \\
\text { Arrest, CKD }\end{array}$ & $\begin{array}{l}\text { Ischemicne- } \\
\text { phrosclerosis }+ \\
\text { Sepsis related } \\
\text { Acute Kidney } \\
\text { Injury }\end{array}$ & $\begin{array}{l}\text { Continuous } \\
\text { Ambulatory } \\
\text { Peritoneal } \\
\text { Dialysis }\end{array}$ & $\begin{array}{l}3 \text { months } \\
\text { CAPD + } 1 \\
\text { month HD }\end{array}$ & IHD & $20 \%$ & Nil & 46 \\
\hline 13 & 37 & $\mathrm{~F}$ & $\begin{array}{l}\text { CKD/ IGA } \\
\text { nephropathy }\end{array}$ & IGA Nephropathy & $\begin{array}{l}\text { Continuous } \\
\text { Ambulatory } \\
\text { Peritoneal } \\
\text { Dialysis }+ \\
\text { transitioned to } \\
\text { Haemodialysis }\end{array}$ & $\begin{array}{l}36 \text { months } \\
\text { CAPD }+12 \\
\text { month HD }\end{array}$ & $\begin{array}{l}\text { Peripartum } \\
\text { cardiomyop- } \\
\text { athy }\end{array}$ & $15 \%$ & $\begin{array}{l}\text { ECMO } \\
\text { post Tx }\end{array}$ & 28 \\
\hline 14 & 43 & $\mathrm{~F}$ & SLE & Lupus Nephritis & Haemodialysis & $\begin{array}{l}60 \text { months } \\
\text { (Multiple renal } \\
\text { transplantation) }\end{array}$ & IHD & $28 \%$ & Nil & 34 \\
\hline 15 & 16 & M & CKD,IHD & $\begin{array}{l}\text { Obstructive } \\
\text { Uropathy }\end{array}$ & Haemodialysis & $\begin{array}{l}\text { CAPD } 12 \\
\text { months + HD } 9 \\
\text { months }\end{array}$ & $\mathrm{DCM}$ & $10 \%$ & Nil & 38 \\
\hline 16 & 54 & $\mathrm{M}$ & $\begin{array}{l}\text { Chronic AF, } \\
\text { Hyper- } \\
\text { tension, } \\
\text { Dyslipidemia } \\
\text { PVD,Osteo- } \\
\text { porosis }\end{array}$ & $\begin{array}{l}\text { Ischemic } \\
\text { Nephrosclerosis } \\
+/- \text { Cardiorenal } \\
\text { Syndrome }\end{array}$ & $\begin{array}{l}\text { Continuous } \\
\text { Ambulatory } \\
\text { Peritoneal } \\
\text { Dialysis }\end{array}$ & 12 months & $\mathrm{DCM}$ & $18 \%$ & Nil & 30 \\
\hline
\end{tabular}

DCM: Dilated Cardiomyopathy. AF: Atrial Fibrillation. MI: Myocardial Infarct. CKD: Chronic Kidney Disease. CAPD: Continuous Ambulatory Peritoneal Dialysis. HD: Haemodialysis. MCG: Mesangiocapillary Glomerulonephritis. WAGR: Wilms Tumors, Aniridia, Genitourinary Abnormality, Mental Retardation syndrome. RF: Rheumatic Fever. ECMO: Extracorporeal Membrane Oxygenation. IABP: Intraortic Balloon Pump. Tx: Transplant, Strep: Streptococcal

Table 2: Patient Characteristics Pre Transplant

\begin{tabular}{|c|c|c|c|}
\hline Patient characteristics Pre-Transplant & Mean \pm SD & Range & Percentage $\%$ \\
\hline Age at transplantation (y) & $42.875 \pm 13.0$ & $16-61$ & \\
\hline Male sex $(13 / 16)$ & & & 81 \\
\hline Dilated Cardiomyopathy (8/16) & & & 50 \\
\hline Ischemic Cardiomyopathy 3 (18.75\%) & & & 18.75 \\
\hline NYHA class III (6/16) & & & 37.5 \\
\hline NYHA class IV (10/16) & & & 62.5 \\
\hline IABP/ECMO Peri- Transplant (5/16) & & & 31.3 \\
\hline LVEF (\%) & $18.9 \pm 5.7$ & & \\
\hline Mean Pulmonary Artery Pressure (mmHg) & $36.7 \pm 9.7$ & $13-50$ & \\
\hline
\end{tabular}




\begin{tabular}{|l|c|c|c|}
\hline Ischemic Nephrosclerosis/ Cardiorenal Syndrome (4/16) & & & 25 \\
\hline Glomerulonephritis (4/16) & & & \\
\hline Iga Nephropathy (3/16) & & & \\
\hline Creatinine level (mmol/L) & $390 \pm 179.2$ & \\
\hline RRT prior to Transplantation (14/16) & & & \\
\hline Time on RRT prior to transplant (months) & $36.4 \pm 29.5$ & & \\
\hline Wait time to transplant (months) & $12.2 \pm 7.1$ & & \\
\hline
\end{tabular}

The patient selection criteria include all patients with New York Heart Association class III and IV cardiac failure as per the International Society for Heart and Lung Transplant (ISHLT) indications for cardiac transplantation. The patients must have had concomitant renal failure with creatinine clearances of less than $30 \mathrm{~mL} / \mathrm{min}$ and/or required renal replacement therapy at time of consideration for cardiac transplantation.

HLA typing was not done preoperatively on all patients and was omitted for the patients that did have them performed. The hearts were procured by the cardiac transplantation service at our institution, and the kidneys by the surgical team designated by the organ procurement coordinator. The heart transplant was performed first on table followed by kidney transplantation in all 16 cases.

Immunosuppressive therapy was documented and tabulated and changes to their regimen in the 25 year follow up period were documented. Endomyocardial biopsies were performed according to our institutions protocol or when cardiac rejection was suspected the grading was documented and tabulated (Table 3) as per the ISHLT classification. Only the most significant episode of rejection was documented. Renal rejection was confirmed with renal biopsies when clinically and biochemically suspected and rejection episodes tabulated (Table 3).

Table 3: Acute Complications Post Transplant and Episodes of Rejection with Immunosuppressive Regimen

\begin{tabular}{|c|c|c|c|c|c|}
\hline Patient & $\begin{array}{l}\text { Acute } \\
\text { complications }\end{array}$ & $\begin{array}{l}\text { Cardiac } \\
\text { rejection }\end{array}$ & \begin{tabular}{c|} 
Renal \\
Rejection
\end{tabular} & $\begin{array}{l}\text { Immunosuppression Post } \\
\text { Transplant }\end{array}$ & Current immunosuppresion \\
\hline 1 & $\begin{array}{l}\text { Sepsis from sternal wound } \\
\text { infection- Resuting in Acute } \\
\text { Renal failure }\end{array}$ & $\begin{array}{l}\text { 3A rejection. } \\
\text { Pulsed } \\
\text { methylpred }\end{array}$ & Nil & $\begin{array}{l}\text { Cyclosporine } 100 \mathrm{mg} \text { bd } \\
\text { Mycophenolate } 500 \mathrm{mg} \text { bd } \\
\text { Prednisone } 10 \mathrm{mg} \text { daily }\end{array}$ & $\begin{array}{l}\text { Cyclosporine } 100 \mathrm{mg} \text { bd } \\
\text { Mycophenolate } 500 \mathrm{mg} \text { bd } \\
\text { Prednisone } 10 \mathrm{mg} \text { daily }\end{array}$ \\
\hline 2 & Sepsis VRE, Pericardial Effusion & Nil & Yes & $\begin{array}{l}\text { Cyclosporine } 125 \mathrm{mg} \text { bd, } \\
\text { Mycophenolate1g BD, } \\
\text { Prednisone } 10 \mathrm{mg}\end{array}$ & $\begin{array}{l}\text { Tacrolimus } 4 \mathrm{mg} \text { tds, Sirolimus } 1 \\
\text { mg tds, Mycophenolate, } 1.5 \mathrm{~g} \text { bd, } \\
\text { Prednisone } 25 \mathrm{mg} \text { daily }\end{array}$ \\
\hline 3 & $\begin{array}{l}\text { Cardiac Arrest- Resuscitated, } \\
\text { ARF- HD, Leakage from bladder } \\
\text { anastomosis, Herpes Simplex } \\
\text { infection }\end{array}$ & $\begin{array}{l}3 \text { A rejection. } \\
\text { Pulsed } \\
\text { methylpred }\end{array}$ & Nil & $\begin{array}{l}\text { Cyclosporine } 150 \mathrm{mgbd} \\
\text { Mycophenolate } 1 \mathrm{~g} \mathrm{bd} \\
\text { Pred } 15 \mathrm{mg} \text { bd }\end{array}$ & $\begin{array}{l}\text { Tacrolimus } 2 \mathrm{mg} \text { bd } \\
\text { Mycophenolate } 1 \mathrm{~g} \text { bd } \\
\text { Prednisone } 15 \mathrm{mg} \text { bd }\end{array}$ \\
\hline 4 & Stroke post transplant & Nil & Nil & $\begin{array}{l}\text { Cyclosporine } 100 \mathrm{mg} \text { bd } \\
\text { Azathioprine } 150 \mathrm{mg} \text { daily } \\
\text { Prednisone } 25 \mathrm{mg} \text { daily }\end{array}$ & $\begin{array}{l}\text { Tacrolimus } 2 \text { mg bd Everolimus } \\
0.75 \mathrm{mg} \text { bd Mycophenolate } 500 \\
\text { mg bd Prednisone } 2.5 \mathrm{mg} \text { daily }\end{array}$ \\
\hline 5 & Nil Significant & Nil & Nil & \begin{tabular}{|l|} 
Cyclosporin $150 \mathrm{mg}$ bd \\
Azathioprine $100 \mathrm{mg}$ daily \\
Prednisone 12.5 daily
\end{tabular} & $\begin{array}{l}\text { Cyclosporin } 100 \mathrm{mg} \text { daily } \\
\text { Everolimus } 0.75 \mathrm{mg} \text { bd } \\
\text { Prednisone } 5 \mathrm{mg} \text { daily }\end{array}$ \\
\hline 6 & $\begin{array}{l}\text { Cardiac Arrest off bypass. Resus- } \\
\text { citated successfully }\end{array}$ & 1a rejection & Nil & \begin{tabular}{|l|} 
Cyclosporin $250 \mathrm{mg}$ bd \\
Azathioprine $125 \mathrm{mg}$ daily \\
Prednisone $25 \mathrm{mg}$ daily
\end{tabular} & $\begin{array}{l}\text { cyclosporin } 100 \mathrm{mg} \text { bd, } \\
\text { Azathioprine } 75 \mathrm{mg} \text { daily } \\
\text { prednisone } 10 \mathrm{mg} \text { faily }\end{array}$ \\
\hline 7 & Nil Significant & Nil & Nil & $\begin{array}{l}\text { Cyclosporin } 25 \mathrm{mg} \text { Bd } \\
\text { Everolimus } 0.5 \mathrm{mg} \text { mane } \\
\text { Mycophenolate } 1 \mathrm{gbd} \\
\text { Prednisone } 5 \mathrm{mg} \text { daily }\end{array}$ & $\begin{array}{l}\text { Cyclosporin } 25 \mathrm{mg} \mathrm{Bd} \\
\text { Everolimus } 0.5 \mathrm{mg} \text { mane } \\
\text { Mycophenolate } 1 \mathrm{gbd} \\
\text { Prednisone } 5 \mathrm{mg} \text { daily }\end{array}$ \\
\hline 8 & $\begin{array}{l}\text { VA ecmo post tx-Respiratory } \\
\text { Failure- re intubated, sepsis } \\
\text { (lung),ATN requiring CV- } \\
\text { VHDF-HD post tx,DIC, Ischemic } \\
\text { hepatitis, GI bleeding }\end{array}$ & Nil & Nil & $\begin{array}{l}\text { Cyclosporin } 150 \mathrm{mg} \text { bd } \\
\text { Azathioprine } 100 \mathrm{mg} \text { daily } \\
\text { Prednisone } 12.5 \text { daily }\end{array}$ & $\begin{array}{l}\text { Cyclosporin } 100 / 75 \\
\text { Mycophenolate } 1.5 \mathrm{~g} \mathrm{BD} \\
\text { Prednisolone } 10 \mathrm{mg} \text { daily }\end{array}$ \\
\hline 9 & Nil Significant & $\begin{array}{l}\text { 3A rejection. } \\
\text { Methylpredni- } \\
\text { sone }\end{array}$ & Nil & $\begin{array}{l}\text { Cyclosporine } 100 \mathrm{mg} \text { bd } \\
\text { Azathioprine } 150 \mathrm{mg} \text { daily } \\
\text { Prednisone } 10 \mathrm{mg}\end{array}$ & $\begin{array}{l}\text { Cyclosporin } 75 / 50 \\
\text { Mycophenolate } 1 \mathrm{~g} \text { bd } \\
\text { Pred } 3 \mathrm{mg} \text { daily }\end{array}$ \\
\hline 10 & Ureteric leak- HD post op & $\begin{array}{l}\text { Mild 1a } \\
\text { rejection }\end{array}$ & Nil & $\begin{array}{l}\text { Cyclosporine } 100 \mathrm{mg} \text { bd } \\
\text { Azathioprine } 150 \mathrm{mg} \text { daily } \\
\text { Prednisone } 10 \mathrm{mg}\end{array}$ & $\begin{array}{l}\text { Cyclosporin } 75 / 50 \\
\text { Mycophenolate } 1 \mathrm{~g} \text { bd } \\
\text { Pred } 3 \mathrm{mg} \text { daily }\end{array}$ \\
\hline
\end{tabular}




\begin{tabular}{|c|c|c|c|c|c|}
\hline 11 & Nil Significant & Mild & Nil & $\begin{array}{l}\text { Cyclosporin } 100 / 75 \mathrm{Aza}- \\
\text { thioprine } 150 \mathrm{mg} \text { daily, } \\
\text { Prednisolone } 10 \mathrm{mg} \text { daily }\end{array}$ & $\begin{array}{l}\text { Cyclosporin } 100 / 75 \\
\text { Mycophenolate } 1.5 \mathrm{~g} \text { BD } \\
\text { Prednisolone } 10 \mathrm{mg} \text { daily }\end{array}$ \\
\hline 12 & $\begin{array}{l}\text { Renal Biopsy hemorrhage- } \\
\text { laparotomy. CVVHDF, } \\
\text { pseudobstruction }\end{array}$ & Nil & Nil & $\begin{array}{l}\text { Cyclosporin } 125 \mathrm{mg} \text { bd } \\
\text { Mycophenolate } 1 \mathrm{~g} \mathrm{Bd} \\
\text { Prednisolone } 15 \mathrm{mg} \text { daily }\end{array}$ & $\begin{array}{l}\text { Cyclosporin } 50 \mathrm{mg} \text { bd } \\
\text { Mycophenolate } 1 \mathrm{gbd} \\
\text { Sirolimus } 1 \mathrm{mg} \text { daily }\end{array}$ \\
\hline 13 & Cardiogenic shock, Pneumothorax & $\begin{array}{l}\text { 3a Rejection. } \\
\text { Plasmapheresis }+ \\
\text { methylpredni- } \\
\text { sone }\end{array}$ & Yes & $\begin{array}{l}\text { Cyclosporine } 125 \mathrm{mg} \text { bd } \\
\text { Azathioprine } 150 \mathrm{mg} \text { daily } \\
\text { prednisolone } 50 \mathrm{mg} \text { daily }\end{array}$ & $\begin{array}{l}\text { Tacrolimus } 5 \mathrm{mg} \text { bd, } \\
\text { Azathioprine } 100 \mathrm{mg} \text { daily, } \\
\text { Prednisone } 7 \mathrm{mg} \text { daily }\end{array}$ \\
\hline 14 & Sepsis, hypotension & $3 \mathrm{~b}$ rejection & Nil & $\begin{array}{l}\text { Cyclosporine } 150 \mathrm{mg} \text { bd } \\
\text { Azathioprine } 100 \mathrm{mg} \text { daily } \\
\text { Prednisone } 12.5 \mathrm{mg} \text { daily }\end{array}$ & $\begin{array}{l}\text { Cyclosporin } 100 / 75 \\
\text { Mycophenolate } 1.5 \mathrm{~g} \text { BD } \\
\text { Prednisolone } 10 \mathrm{mg} \text { daily }\end{array}$ \\
\hline 15 & Nil Significant & $3 \mathrm{~b}$ rejection & Yes & $\begin{array}{l}\text { Cyclosporine } 150 \mathrm{mg} \text { bd } \\
\text { Azathioprine } 125 \mathrm{mg} \text { daily } \\
\text { Prednisolone } 25 \mathrm{mg} \text { daily }\end{array}$ & $\begin{array}{l}\text { Tacrolimus } 5 \mathrm{mg} \text { daily Azathio- } \\
\text { prine } 150 \mathrm{mg} \text { daily Mycophenolate } \\
500 \mathrm{mg} \text { bd Prednisone } 15 \mathrm{mg} \text { daily, }\end{array}$ \\
\hline 16 & $\begin{array}{l}\text { Respiratory Failure- re intubat- } \\
\text { ed, sepsis (lung), ATN requiring } \\
\text { CVVHDF-HD post tx } 1 / 12\end{array}$ & 3a Rejection & Nil & $\begin{array}{l}\text { Cyclosporine } 150 \mathrm{mg} \text { bd } \\
\text { Mycophenolate } 1.5 \mathrm{gm} \text { bd } \\
\text { Prednisone } 25 \mathrm{mg} \text { daily }\end{array}$ & $\begin{array}{l}\text { Cyclosporin } 100 \mathrm{mg} \text { daily Everoli- } \\
\text { mus } 0.75 / 0.25 \mathrm{Mycophenolate} 2 \mathrm{~g} \\
\text { bd Prednisone } 2.5 \mathrm{mg} \text { daily }\end{array}$ \\
\hline
\end{tabular}

Cumulative survival outcomes were calculated with the actuarial method of estimation. The actuarial survival was calculated for the combined heart and kidney transplant group, the cardiac transplant only group with a subsequent comparison over a 25 year follow up period. These results were graphically analysed and compare

\section{Results}

\section{Pre-transplant characteristics}

Patient demographic and clinical characteristics are indicated in Table 1. The mean and standard deviations of preoperative characteristics are indicated in Table 2 . The mean age of the recipients was 42.9 with a range of 16 - 61 . The majority of recipients were of Caucasian decent however there were 2 of Asian descent and 2 of Mediterranean descent. Thirteen of the recipients were male and three were female. $50 \%$ of the recipients had dilated cardiomyopathy as their primary cardiac pathology whilst ischemic nephrosclerosis or glomerulonephritis encompassed the majority of primary renal pathology at $50 \%$. Five patients or $33 \%$ required ECMO or IABP prior to combine transplantation. The mean preoperative ejection fraction was 18.9 percent with a range of $10-30$ percent. The mean pulmonary arterial pressure was $36.7 \mathrm{mmHg}$ with a range of $13-50$. Three patients were recipients of multiple organ transplantation and 1 of these patients was a recipient of sequential heart and kidney transplant followed by combined heart kidney transplant due to allograft failure.

\section{Postoperative outcomes}

There was no operative mortality in all 16 cases. The acute complications post transplant along with their initial immunosuppressant regimens are listed in Table 3. Their initial immunosuppressant regimen and their subsequent changes are also listed in Table 3.

One patient died within the first month of combined heart transplantation from multisystem organ dysfunction and sepsis. Sepsis from a respiratory source was the most common post operative complication with $31 \%$ of patients having a documented episode of significant respiratory sepsis. Two of these patients required re-intubation to support their respiratory function. Five patients or $31 \%$ required a period of renal replacement therapy post operatively. All except 1 patient was transitioned off renal replacement within the first 6 months post transplant.

There were 7 patients or $43 \%$ who had significant cardiac rejection with 2 patients experiencing mild cardiac rejection as per the ISHLT classification. Three or $19 \%$ of patients had documented renal rejection. Two of these patients did have a preceding significant episode cardiac rejection with only one patient having and isolated episode of renal rejection.

Renal graft function remained stable throughout the study period in 14 out of 16 patients or $87.5 \%$. 11 out of 16 patients had stable cardiac function post combined transplant throughout the study period or $69 \%$ of the cohort.

Chronic allograft vasculopathy was the most common major complication in the study period with $25 \%$ of patients experiencing reduced cardiac function as a consequence.

The actuarial 1 year survival in the combined transplant group was $75 \%$ with 4 out of 16 dying within the first year (25\%). In comparison the actuarial survival of the heart only transplant group was $86 \%$ accounting for 116 deaths within the first year of the 866 patients in this group over a 25 year period.

Actuarial survival at 5, 10, 15 and 25 years for the combined transplant group was $69 \%, 55 \%, 43.7 \%$ and $43.7 \%$ respectively. (Figure 1) 


\section{Cumulative survival Combined Heart Kidney}

Tx

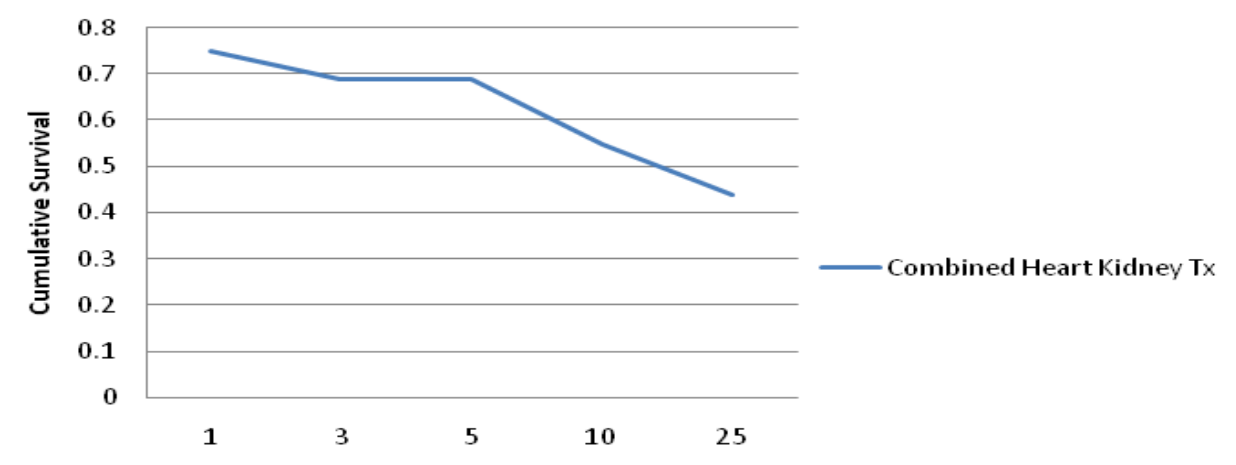

FIgure 1: Cumulative Survival of Combined Heart Kidney Transplant over 25 years.

In comparison cumulative survival of the heart only transplant group at the 5, 10, 15, and 25 year mark was $76 \%, 59 \%$, 45\%, and $23 \%$ respectively. (Figure 2 )

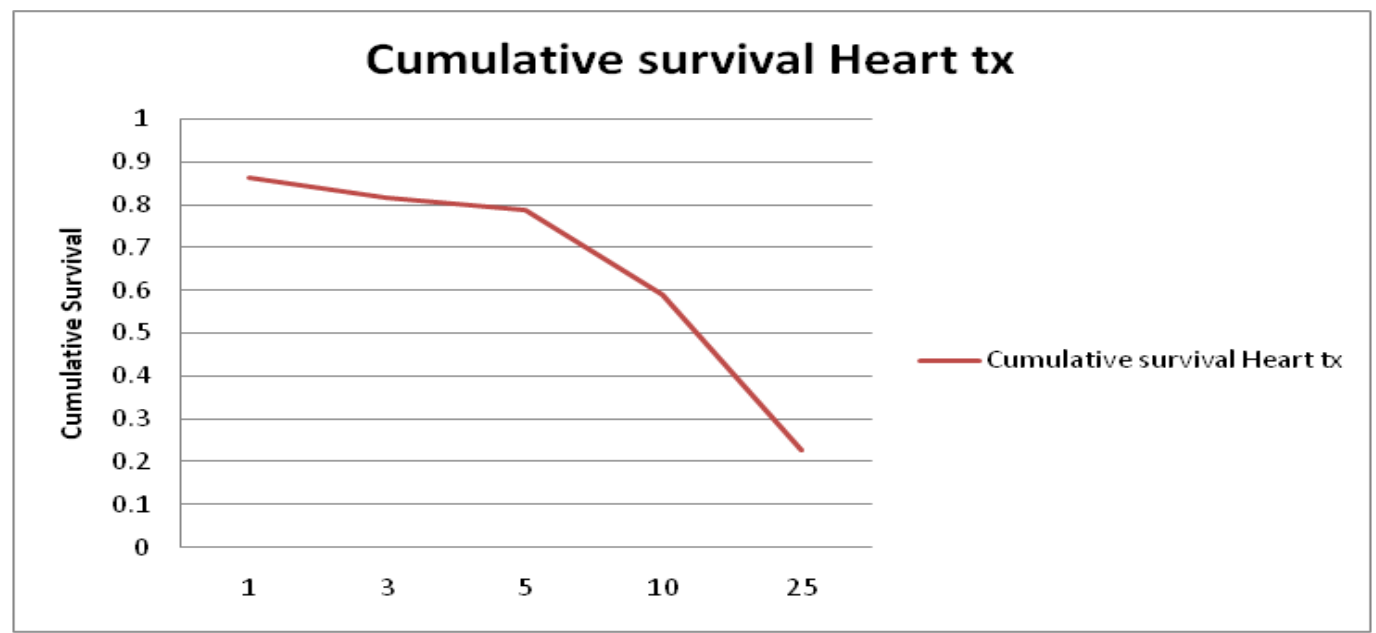

Figure 2: Cumulative Survival of Solitary Heart Transplant over 25 years.

The actuarial survival of the two groups over a 25 year period was then compared. (Figure 3) At the 10 year mark, actuarial survival for the combined group was comparable to the heart only transplant group with figures of 55\% versus $59 \%$.

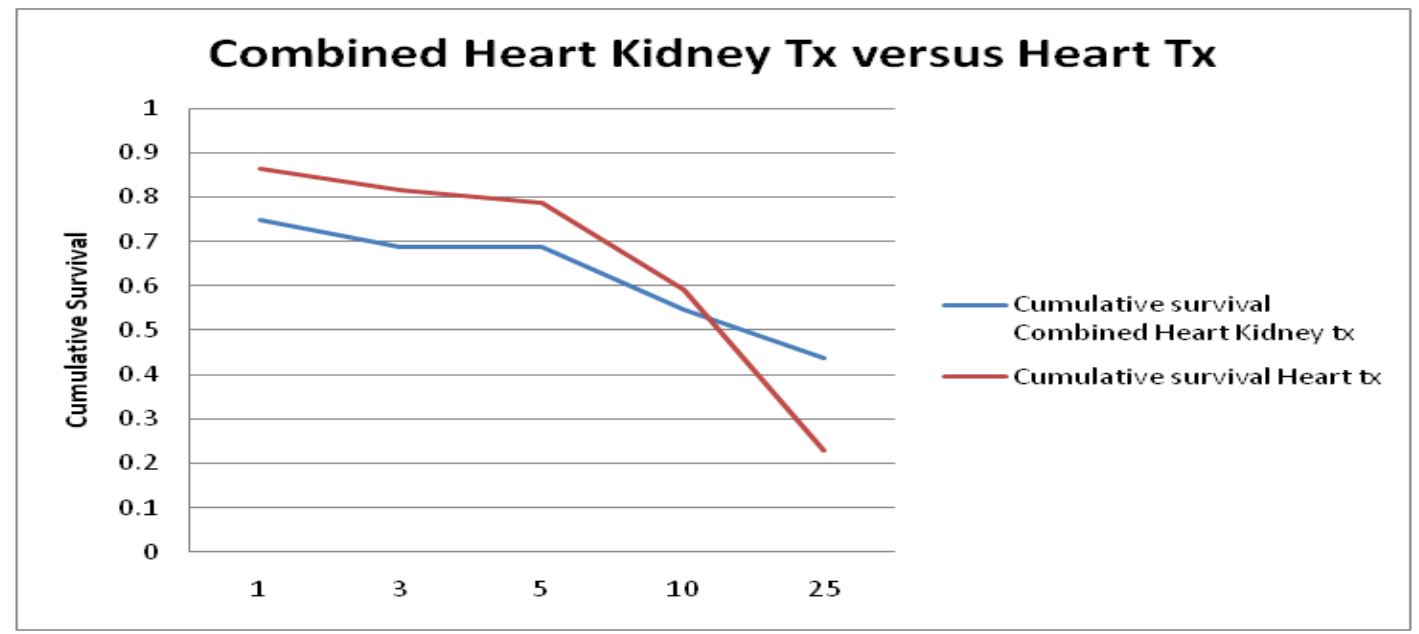

Figure 3: Combined Heart Kidney Transplant Cumulative Survival Versus Solitary Heart Transplant Cumulative Survival over 25 years.

The main causes of death were sepsis resulting in multisystem organ failure, cardiac arrest and malignancy. There was a low incidence of dual graft failure as the primary cause of death. Most patients after the first year post combined procedure had a relatively good graft longevity and function. 


\section{Discussion}

The current study represents the longest single center experience of simultaneous combined heart kidney transplantation. It confirms and demonstrates that combined heart kidney transplantation has favourable short and long term outcomes for patients with end stage cardiac and renal failure especially when on renal replacement therapy at the time of transplantation which often is a contraindication in itself to solitary organ transplant.

The overwhelming majority of our patients were on renal replacement therapy at the time of their combined heart and kidney transplantation. Although there is conflicting analysis of registry data by Russo et al suggesting greater adverse outcomes of patients who underwent combined heart kidney transplantation whilst on renal replacement therapy in comparison to those not on renal replacement therapy with an EGFR of less than 30, our study showed markedly improved survival outcomes in 14 out of 16 of our patients or $87.5 \%$ of the cohort (Table 4 ). This emphasizes the superior outcomes for patients with concurrent end stage cardiac and renal failure requiring renal replacement therapy who undergoes combined cardiac and renal transplantation.

Table 4: Long Term Graft Function and Complications Post Transplantation with Survival Outcomes and Cause of Mortality

\begin{tabular}{|c|c|c|c|c|c|c|c|}
\hline Patient & $\begin{array}{l}\text { Renal Graft } \\
\text { function- Cre- } \\
\text { atinine } \mathrm{mmol} / \mathrm{l} \\
\end{array}$ & $\begin{array}{l}\text { Cardiac Graft } \\
\text { function: Ejec- } \\
\text { tion fraction }\end{array}$ & $\begin{array}{l}\text { Major Long term complica- } \\
\text { tions post transplantation }\end{array}$ & $\begin{array}{l}\text { MinorCom- } \\
\text { plications post } \\
\text { transplantation }\end{array}$ & $\begin{array}{l}\text { Graft } \\
\text { survival }\end{array}$ & $\begin{array}{l}\text { Patient } \\
\text { survival }\end{array}$ & $\begin{array}{l}\text { Cause } \\
\text { of Death }\end{array}$ \\
\hline 1 & 100 & $50 \%$ & 1. CMV gastritis/ esophagitis & $\begin{array}{l}\text { Squamous Cell } \\
\text { Skin Cancer }\end{array}$ & $\begin{array}{l}\text { Both } \\
\text { Functional }\end{array}$ & Alive & N/A \\
\hline 2 & $\begin{array}{l}\text { Graft failure- } \\
\text { Rejection Back } \\
\text { on HD }\end{array}$ & $\begin{array}{l}\text { AMI-Cardiac } \\
\text { graft failure }\end{array}$ & $\begin{array}{l}\text { CMV pneumonitis, } \\
\text { Recurrent VRE urosepsis }\end{array}$ & N/A & $\begin{array}{l}\text { Both grafts } \\
\text { failed }\end{array}$ & Deceased & Cardiac Arrest \\
\hline 3 & 159 & $42 \%$ & Allograft vasculopathy & N/A & $\begin{array}{l}\text { Both } \\
\text { Functional }\end{array}$ & Deceased & Cardiac Arrest \\
\hline 4 & 125 & $50 \%$ & Nil & N/A & $\begin{array}{l}\text { Both } \\
\text { Functional }\end{array}$ & Alive & $\mathrm{N} / \mathrm{A}$ \\
\hline 5 & 120 & $60 \%$ & $\begin{array}{l}\text { 1. Recurrent infections: Staph, } \\
\text { CMV, HSV } \\
\text { 2. Aortic Dilatation: Bentall's } \\
\text { CABG } 04 \\
\text { 3. Allograft Vasculopathy }\end{array}$ & N/A & $\begin{array}{l}\text { Both } \\
\text { Functional }\end{array}$ & Alive & $\mathrm{N} / \mathrm{A}$ \\
\hline 6 & 130 & $60 \%$ & Allograft vasculopathy & $\begin{array}{l}\text { Squamous Cell } \\
\text { Skin Cancer/ } \\
\text { Bowens disease }\end{array}$ & $\begin{array}{l}\text { Cardiac } \\
\text { Graft } \\
\text { Failed }\end{array}$ & Deceased & $\begin{array}{l}\text { Allograft } \\
\text { vasculopathy }\end{array}$ \\
\hline 7 & 135 & $25 \%$ & $\begin{array}{l}\text { 1. Atrial Myxoma/ Atrial } \\
\text { Thrombus } \\
\text { 2. Subdural Haemorrhage }\end{array}$ & N/A & $\begin{array}{l}\text { Both Func- } \\
\text { tional }\end{array}$ & Alive & N/A \\
\hline 8 & 110 & $25 \%$ & Nil & N/A & $\begin{array}{l}\text { Cardiac } \\
\text { Graft } \\
\text { Failed }\end{array}$ & Deceased & $\begin{array}{l}\text { Multisystem } \\
\text { organ failure in } \\
\text { ICU post Tx }\end{array}$ \\
\hline 9 & 90 & $50 \%$ & $\begin{array}{l}\text { SCC- excision required. Mandib- } \\
\text { ular excision }\end{array}$ & $\begin{array}{l}\text { HPT, Dyslip- } \\
\text { idemia, Gout, } \\
\text { osteoporosis }\end{array}$ & $\begin{array}{l}\text { Both } \\
\text { Functional }\end{array}$ & Alive & N/A \\
\hline 10 & 77 & $50 \%$ & $\begin{array}{l}\text { Malignancy - Non Lymphoprolif- } \\
\text { erative/Disseminated adenocarci- } \\
\text { noma CMV gastritis/ disseminat- } \\
\text { ed herpez zoster }\end{array}$ & N/A & $\begin{array}{l}\text { Both } \\
\text { Functional }\end{array}$ & Deceased & $\begin{array}{l}\text { Malignancy - } \\
\text { Non Lymph- } \\
\text { oproliferative } \\
\text { malignancy }\end{array}$ \\
\hline 11 & 121 & $50 \%$ & Nil & $\begin{array}{l}\text { Basal Cell Skin } \\
\text { Cancer / Bowens } \\
\text { disease }\end{array}$ & $\begin{array}{l}\text { Both } \\
\text { Functional }\end{array}$ & Alive & $\mathrm{N} / \mathrm{A}$ \\
\hline 12 & 105 & $60 \%$ & Nil & N/A & $\begin{array}{l}\text { Both } \mathrm{f} \\
\text { unctional }\end{array}$ & Alive & $\mathrm{N} / \mathrm{A}$ \\
\hline 13 & 80 & $35 \%$ & $\begin{array}{l}\text { P.E, Lymphoproliferative } \\
\text { disorder((PTLD) }\end{array}$ & N/A & $\begin{array}{l}\text { Both } \\
\text { Functional }\end{array}$ & Deceased & $\begin{array}{l}\text { Lymphoprolif- } \\
\text { erative disorder }\end{array}$ \\
\hline 14 & N/A & N/A & Sepsis & N/A & $\begin{array}{l}\text { Both } \\
\text { Functional }\end{array}$ & Deceased & $\begin{array}{l}\text { Sepsis }+ \text { multi- } \\
\text { system organ } \\
\text { failure }\end{array}$ \\
\hline 15 & 220 & $28 \%$ & Nil & N/A & $\begin{array}{l}\text { Cardiac } \\
\text { Graft } \\
\text { Failed }\end{array}$ & Deceased & Graft failure \\
\hline 16 & 135 & $50 \%$ & Allograft Vasculopathy & $\begin{array}{l}\text { Squamous Cell } \\
\text { Skin Cancer }\end{array}$ & $\begin{array}{l}\text { Both } \\
\text { Functional }\end{array}$ & Alive & N/A \\
\hline
\end{tabular}


CMV: Cytomegalovirus, HSV: Herpes Simplex Virus, CABG: Coronary Artery Bypass Graft. HPT: Hypertension. Staph: Staphylococcus. ICU: Intensive Care Unit.

Our study showed that kidney allograft function in the setting of combined heart kidney transplantation remained relatively intact with 15 out of 16 patients experiencing no renal graft failure. Simultaneous graft failure leading to death was observed in 1 patient in our study. This lower incidence of renal rejection was also reported in several other studies ${ }^{[12,13,29]}$. The overall rate of rejection was encouragingly low with the majority of patients experiencing more significant cardiac rejection episodes that responded to either intravenous pulse steroids with one patient requiring plasmapheresis. Why this immunological tolerance was observed in the donor kidney is potentially multi-factorial with more stringent monitoring for cardiac rejection and the previously proposed phenomena of immunogenic tolerance to the less antigenic organ in the context of combined transplantation ${ }^{[29-36]}$.

Most importantly given the 1,5,10, and 25 year actuarial survival of $75 \%, 69 \%, 55 \%$ and $43.7 \%$ respectively, combined heart kidney transplantation can be viewed as a viable option for patients with concurrent end stage cardiac and renal dysfunction ${ }^{[37]}$. Our study correlates closely with various other single center studies (Table 5) that have also demonstrated excellent short and long term survival outcomes of combined heart kidney transplantation over significant time periods.

Table 5: Survival Rate and Duration of Follow Up of Combined Heart Kidney Transplantation in a Single Center.

\begin{tabular}{|l|c|c|c|l|}
\hline Studies & 1 year survival \% & 5 year survival \% & Number of Patients & Duration of Follow Up \\
\hline Raichlin et al $^{[15]}$ & 83 & 83 & 12 & 10 years \\
\hline Luckraz et al $^{[19]}$ & 77 & 60 & 13 & 15 years \\
\hline Vermes et al $^{[16]}$ & 66 & 55 & 12 & 10 years \\
\hline Trachiotis et al $^{[12]}$ & 87 & 87 & 8 & 8 years \\
\hline Leesera et al $^{[17]}$ & 77 & 60 & 13 & 10 years \\
\hline Ruzza et al $^{[35]}$ & 90 & 70 & 30 & Ruzza et al 35 \\
\hline
\end{tabular}

The challenge to combined heart kidney transplantation is the rationality and ethical dilemma surrounding combined organ transplantation given the limitations and constraints surrounding donor organ availability. In most cases multiple organs from either a single or multiple donors will be transplanted into a single recipient, posing a significant burden on the already limited resources of the state-wide and national transplant service. The overall waitlist times for combined heart kidney transplantation with a mean of 12.1 months in our study offers further emphasizes that this is a viable option with acceptable waiting times for patients with dual heart and kidney failure.

\section{Conclusion}

In conclusion, the results of this study further emphasizes that combined heart kidney transplantation produces good short and long term outcomes for patients with end stage heart and kidney failure and especially patients with end stage heart failure on renal replacement therapy. These favourable survival outcomes and lower rates of organ rejection with combined transplantation has now been reproduced consistently from multiple international centers with different selection criteria, making combined heart kidney transplantation a viable and effective therapeutic option for patients with end stage cardiac and renal failure who would previously not have qualified for solitary organ transplantation.

Conflicts of Interest: No disclosures in the submission of this work.

\section{References}

1. McGiffin, D.C., Kirklin, J.K., Naftel, D.C. Acute renal failure after heart transplantation and cyclosporine therapy. (1985) J Heart Transplant 4(4): 396-399.

2. Taylor, D.O., Stehlik, J., Edwards, L.B., et al. Registry of the International Society for Heart and Lung Transplantation: Twenty sixth Official Adult Heart Transplant Report-2009. (2009) J Heart Lung Transplant 28(10): 1007-1022.

3. McAlister, F., Ezekowitz, J., Tonelli, M., et al. Renal Insufficiency and Heart Failure: Prognostic and Therapeutic Implications From A Prospective Cohort Study. (2004) Circulation 109(8): 1004-1009.

4. McClellan, W.M., Flanders, W.D., Langston, R.D., et al. Anemia and renal insufficiency are independent risk factors for death among patients with congestive heart failure admitted to community hospitals: a population-based study. (2002) J Am Soc Nephrol 13(7): 1928-1936.

5. Dries, D.L., Exner, D.V., Domanski, M.J., et al. The prognostic implications of renal insufficiency in asymptomatic and symptomatic patients with left ventricular systolic dysfunction. (2000) J Am Coll Cardiol 35(3): 681-689.

6. Hillege, H.L., Girbes, A.R., de Kam, P.J., et al. Renal function, neurohormonal activation, and survival in patients with chronic heart failure. (2000) Circulation 102(2): 203-210.

7. Al-Ahmad, A., Rand, W.M., Manjunath, G., et al. Reduced kidney function and anemia as risk factors for mortality in patients with left ventricular dysfunction. (2001) J Am Coll Cardiol 38(4): 955-962.

8. Mahon, N.G., Blackstone, E.H., Francis, G.S., et al. The prognostic value of estimated creatinine clearance alongside functional capacity in 
ambulatory patients with chronic congestive heart failure. (2002) J Am Coll Cardiol 40(6): 1106-1113.

9. Kearney, M.T., Fox, K.A., Lee, A.J., et al. Predicting death due to progressive heart failure in patients with mild-to-moderate chronic heart failure. (2002) J Am Coll Cardiol 40(10): 1801-1808.

10. Luthi, J.C., Flanders, W.D., Burnier, M., et al. Anemia and renal in sufficiency are independent risk factors for death among patients with congestive heart failure admitted to community hospitals: a population-based study. (2006) J Am Soc Nephrol 7: 3.

11. Norman, J.C., Brook, M.I., Cooley, D.A., et al. Total support of the circulation of a patient with post-cardiotomy stone-heart syndrome by a partial artificial heart (ALVAD) for five days followed by heart and kidney transplantation. (1978) Lancet 1(8074): 1125-1127.

12. Trachiotis, G.D., Vega, J.D., Johnston, T.S., et al. Ten-year follow-up in patients with combined heart and kidney transplantation. (2003) J Thorac Cardiovasc Surg 126(6): 2065-2071.

13. Blanche, C., Kamlot, A., Blanche, D., et al. Combined heart-kidney transplantation with single donor allografts. (2001) J Thorac Cardiovasc Surg 122(3): 495-500.

14. Bacchi, G., Potena, L., Mosconi, G., et al. Combined Heart-Kidney Transplantation: A Single Center Experience. (2011) Open Journal of Organ Transplant Surgery 1(2): 14-18.

15. Raichlin, E., Kushwaha, S., Daly, R., et al. Combined heart and kidney transplantation provides an excellent survival and decreases risk of cardiac cellular rejection and coronary allograft vasculopathy. (2011) Transplant Proc 43(5): 1871-1876.

16. Vermes, E., Grimbert, P., Sebbag, L., et al. Long-Term Results of Combined Heart and Kidney Transplantation: A French Multicenter Study. International Society for Heart and Lung Transplantation. (2009) Journal of Heart Lung and Transplant 28(5): 440-445.

17. Leesera, D., Jeevanandamb, V., Furukawaa, S., et al. Simultaneous Heart and Kidney Transplantation in Patients with End-stage Heart and Renal Failure. (2001) Am J Transplant 1(1): 89-92.

18. Vincent, J., Jacquet, L., Squifflet, J.P., et al. Combined heart-kidney transplantation: report on six cases. (1998) Nephrol Dial Transplant 13(3): 723-727.

19. Luckraz, H., Parameshwar, J., Charman, S.C., et al. Short- and long-term outcomes of combined cardiac and renal transplantation with allografts from a single donor. (2003) J Heart Lung Transplant 22(12): 1318-1322.

20. Colucci, V., Quaini, E., Magnani, P., et al. Combined heart and kidney transplantation: An effective therapeutic option -- report of six cases. (1997) Eur J Cardiothorac Surg 12(4): 654-658.

21. Shaw, B.W., Bahnson, H.T., Hardesty, R.L., et al. Combined transplantation of heart and liver. (1985) Ann Surg 202(6): 667-672.

22. Savdie, E., Keogh, A.M., Macdonald, P.S., et al. Simultaneous transplantation of the heart and kidney. (1994) Aust N Z J Med 24(5): 554-560.

23. Gonwa, T.A., Husberg, B.S., Klintmalm, G.B., et al. Simultaneous heart and kidney transplantation: a report of three cases and review of the literature. (1992) J Heart Lung Transplant 11(1 Pt 1): 152-155.

24. Benvenuti, C., Bourgeon, B., Chopin, D., et al. Combined heart and kidney transplantation. (1995) Transplant Proc $27: 1694$.

25. Faggian, G., Bortolotti, U., Stellin, G., et al. Combined heart and kidney transplantation: a case report. (1986) J Heart Transplant 5(6): $480-483$.

26. Livesay, S.A., Rolles, K., Calne, R. Successful simultaneous heart and kidney transplantation using the same donor. (1988) Clin Transplant 2: $1-4$.

27. Cachera, J.P., Abbou, C., Deleuze, P., et al. Combined heart and kidney transplantation using the same donor. (1989) Eur J Cardiothorac Surg 3(2): 169-173.

28. Blanche, C., Kamlot, A., Blanche, D.A., et al. Combined heart-kidney transplantation with single-donor allografts. (2001) J Thorac Cardiovasc Surg 122(3): 495-500.

29. Narula, J., Bennet, L.E., DiSalvo, T., et al. Outcome in recipients of combined heart-kidney transplantation. (1997) Transplantation 63(6): 861-867.

30. Laufer, G., Kocher, A., Grabenwöger, M., et al. Simultaneous heart and kidney transplantation as treatment for end-stage heart and kidney failure. (1997) Transplantation 64(8): 1129-1134.

31. Blanche, C., Valenza, M., Czer, L.S., et al. Combined heart and kidney transplantation with allografts from the same donor. (1994) Ann Thorac Surg 58(4): 1135-1138.

32. Wang, S.S., Chou, N.K., Chi, N.H., et al. Simultaneous heart and kidney transplantation for combined cardiac and renal failure. (2006) Transplant Proc 38(7): 2135-2137.

33. Groetzner, J., Kaczmarek, I., Mueller, M., et al. Freedom from graft vessel disease in heart and combined heart- and kidney-transplanted patients treated with tacrolimus-based immunesuppression. (2005) J Heart Lung Transplant 24(11): 1787-1792.

34. Hermsen, J.L., Nath, D.S., del, Rio, A.M., et al. Combined heart-kidney transplantation: the University of Wisconsin experience. (2007) J Heart Lung Transplant 26(11): 1119-1126.

35. Ruzza, A., Vespignani, R., Czer, L., et al. Long- term Survival after Combined Heart and Kidney Transplantation is Comparible to Heart Transplantation Alone. (2009) Circulation 120: S911.

36. Williams, K.A., Hart, D.N., Fabre, J.W., et al. Distribution and quantitation of HLA-ABC and DR antigens on human kidneys and other tissues. (1980) Transplantation 29(4): 274-279.

37. Russo, M.J., Rana, A., Chen, J.M. et al. Pretransplantation patient characteristics and survival following combined heart and kidney transplantation: an analysis of the United Network for Organ Sharing Database. (2009) Arch Surg 144(3): 241-246.

Journal ISSN: 2378-6914 (online)

Journal Title: Journal of Heart and Cardiology

Short title : J Heart Cardiol
Ommega Online Publishers

E-mail: cardiology@ommegaonline.com

Website: www.ommegaonline.org 